\title{
Multi-loop Feynman Integrals and Conformal Quantum Mechanics
}

\author{
A.P. Isaev * \\ Bogoliubov Laboratory of Theoretical Physics, JINR, Dubna, \\ 141 980, Moscow Region, Russia
}

\begin{abstract}
New algebraic approach to analytical calculations of $D$-dimensional integrals for multi-loop Feynman diagrams is proposed. We show that the known analytical methods of evaluation of multi-loop Feynman integrals, such as integration by parts and star-triangle relation methods, can be drastically simplified by using this algebraic approach. To demonstrate the advantages of the algebraic method of analytical evaluation of multi-loop Feynman diagrams, we calculate ladder diagrams for the massless $\phi^{3}$ theory. Using our algebraic approach we show that the problem of evaluation of special classes of Feynman diagrams reduces to the calculation of the Green functions for specific quantum mechanical problems. In particular, the integrals for ladder massless diagrams in the $\phi^{3}$ scalar field theory are given by the Green function for the conformal quantum mechanics.
\end{abstract}

\section{To the memory of Sergei Gorishnii $1958-1988$}

\section{Introduction}

It is well known that the evaluation of the multiple integrals associated with the Feynman diagrams is the main source of physical data in the perturbative quantum field theory. Since the number of diagrams grows enormously in higher orders of perturbation theory, the numerical calculations of the integrals for multi-loop Feynman diagrams are not sufficient to obtain results with desirable precision. That is why analytical calculations of the Feynman diagrams (integrals) start to be important.

For last few years considerable progress was achieved in analytical calculations of multi-loop Feynman integrals (see e.g. [1] - [6] and references therein). It is interesting that in many cases analytical results for the Feynman integrals are expressed in terms of the multiple zeta values and polylogarithms. Note that the multiple zeta values and polylogarithms are very interesting and promising subjects for investigations in modern mathematics (see e.g. [7], [8]).

\footnotetext{
*e-mail address: isaevap@thsun1.jinr.ru
} 
The analytical evaluations of the multi-loop Feynman integrals are usually based on such powerful methods as the integration by parts [9] and star-triangle (uniqueness) relation (see [10], [11] and references therein) methods. These methods have a long history. For example, the star-triangle relations have firstly been considered in the framework of the conformal field theories [12]. Then it was noticed [13] that the star-triangle relation is a kind of the Yang-Baxter equation (see also [14]). In [13], this fact was used to calculate the "fishing-net" Feynman diagram (of a sufficiently large order) for the four-dimensional $(D=4) \phi^{4}$ theory (as well as "triangle-net" and "honey-comb" diagrams for the $\phi^{6}(D=3)$ and $\phi^{3}(D=6)$ theories, respectively).

In this paper a new algebraic approach to analytical calculations of the massless and dimensionally regularized Feynman integrals, e.g., needed for the renormalization group calculations, is developed. In particular, this method is based on using the integration by parts and star-triangle (uniqueness) relation methods. The advantage of our approach is that we change the manipulations with integrals by the manipulations with the algebraic expressions. This drastically simplifies all calculations, as it will be demonstrated by some examples. In particular, we calculate the integrals for ladder Feynman diagrams arising in the $\phi^{3}$ field theory for scalar massless particles. The integrals for these diagrams contribute to many important physical quantities and have been extensively used in many applications, e.g. in the calculations of the conformal four-point correlators in the $N=4$ supersymmetric Yang-Mills theory [15], [16]. The remarkable fact which we have observed is that the evaluation of the integrals for special classes of Feynman diagrams reduces to the calculation of the Green functions for specific (integrable) quantum mechanical problems. For example, the integrals for ladder massless diagrams in the $\phi^{3}$ scalar field theory are given by the expansion over the coupling constant of the Green function for the D-dimensional conformal quantum mechanics.

The paper is organized as follows. In Section 2, we outline the basic concepts of our operator approach. In Section 3, we explain how the integrals for Feynman diagrams can be represented in the operator form. The analytical calculations of the integrals for multi-loop ladder diagrams are presented in Section 4. In Section 4 we also discuss the relation of multi-loop Feynman integrals to Green functions of specific quantum mechanical problems. In Conclusion we discuss possible generalizations and prospects.

\section{Operator Formalism}

Consider the $D$-dimensional Euclidean space $R^{D}$ with the coordinates $x_{i}$, where $i=1, \ldots, D$. We denote $(x p)=\sum_{i} x_{i} p_{i}, x^{2 \alpha}=\left(\sum_{i} x_{i} x_{i}\right)^{\alpha}$ and in general the parameter $\alpha$ is a complex number. Let $\hat{q}_{i}=\hat{q}_{i}^{\dagger}$ and $\hat{p}_{i}=\hat{p}_{i}^{\dagger}$ be operators of coordinate and momentum, respectively,

$$
\left[\hat{q}_{k}, \hat{p}_{j}\right]=i \delta_{k j}
$$

Introduce the coherent states $|x\rangle \equiv\left|\left\{x_{i}\right\}\right\rangle$ and $|k\rangle \equiv\left|\left\{k_{i}\right\}\right\rangle$ which diagonalize the operators of the coordinate and momentum

$$
\hat{q}_{i}|x\rangle=x_{i}|x\rangle, \quad \hat{p}_{i}|k\rangle=k_{i}|k\rangle,
$$


and normalize these states as follows:

$$
\langle x \mid k\rangle=\frac{1}{(2 \pi)^{D / 2}} \exp \left(i k_{j} x_{j}\right), \quad \int d^{D} k|k\rangle\left\langle k\left|=\hat{1}=\int d^{D} x\right| x\right\rangle\langle x| .
$$

We also define the inversion operator $R$

$$
\begin{gathered}
R^{2}=1, \quad R^{\dagger}=R \hat{q}^{2 D}, \quad\langle x| R=\left\langle\frac{1}{x}\right|, \\
R \hat{q}_{i} R=\hat{q}_{i} / \hat{q}^{2}, \quad K_{i}=R \hat{p}_{i} R=\hat{q}^{2} \hat{p}_{i}-2 \hat{q}_{i}(\hat{q} \hat{p}) .
\end{gathered}
$$

where $\frac{1}{x}:=\left\{x_{i} / x^{2}\right\}$.

The well-known formula for the Fourier transformation of the function $1 / k^{2 \beta}$ can be rewritten with the help of eqs. (2) and (3) in the following form:

$$
\left\langle x\left|\frac{1}{\hat{p}^{2 \beta}}\right| y\right\rangle=a(\beta) \frac{1}{(x-y)^{2 \beta^{\prime}}}, \quad\left(a(\beta)=\frac{\Gamma\left(\beta^{\prime}\right)}{\pi^{D / 2} 2^{2 \beta} \Gamma(\beta)}\right) .
$$

where $\beta^{\prime}=D / 2-\beta, \Gamma(\beta)$ is the Euler gamma-function and $\beta^{\prime} \neq 0,-1,-2, \ldots$. Note that $a(\beta)$ obeys the functional equation $a(\beta) a\left(\beta^{\prime}\right)=(2 \pi)^{-D}$. Formula (5) can be interpreted as a definition of the infinite dimensional matrix representation for $\hat{p}^{-2 \beta}$ (the matrix "indices" are the coordinates $x_{i}$ and $y_{i}$ ). In this representation the operator $\hat{q}^{2 \alpha}$ is a diagonal matrix

$$
\left\langle x\left|\hat{q}^{2 \alpha}\right| y\right\rangle=x^{2 \alpha} \delta^{D}(x-y) .
$$

We call the function $1 /(x-y)^{2 \alpha}$ the propagator with the index $\alpha$. Consider the convolution product of two propagators with the indices $\alpha$ and $\beta$ :

$$
\int \frac{d^{D} z}{(x-z)^{2 \alpha}(z-y)^{2 \beta}}=\frac{V\left(\alpha^{\prime}, \beta^{\prime}\right)}{(x-y)^{2(\alpha+\beta-D / 2)}},
$$

where $V(\alpha, \beta)=a(\alpha+\beta) /(a(\alpha) a(\beta))$. This is nothing but the group relation $\hat{p}^{-2 \alpha^{\prime}} \hat{p}^{-2 \beta^{\prime}}=\hat{p}^{-2\left(\alpha^{\prime}+\beta^{\prime}\right)}$ which is written in the matrix form (5).

Using the definition of the inversion operator $R$ (4) one can deduce the main formula

$$
R \hat{p}^{2 \beta} R=\hat{q}^{2\left(\beta+\frac{D}{2}\right)} \hat{p}^{2 \beta} \hat{q}^{2\left(\beta-\frac{D}{2}\right)} .
$$

Then, the group relation $\left(R \hat{p}^{2(\alpha+\beta)} R\right)=\left(R \hat{p}^{2 \alpha} R\right)\left(R \hat{p}^{2 \beta} R\right)$ is equivalent to the identity

$$
\hat{p}^{2 \alpha} \hat{q}^{2 \gamma} \hat{p}^{2 \beta}=\hat{q}^{2 \beta} \hat{p}^{2 \gamma} \hat{q}^{2 \alpha}, \quad(\gamma=\alpha+\beta) .
$$

which can be represented in the form of the commutativity condition $\left[H_{\alpha}, H_{-\beta}\right]=0$ for the operators $H_{\alpha}=\hat{p}^{2 \alpha} \hat{q}^{2 \alpha}$. Thus, $H_{\alpha}$ (for all $\alpha$ ) generate the commutative set of elements in the algebra of functions of $\hat{q}_{i}, \hat{p}_{i}$. Identity (9), represented in the matrix form (5), (6), is the famous star-triangle relation $(\alpha+\beta=\gamma)$ :

$$
\int \frac{d^{D} z}{(x-z)^{2 \alpha^{\prime}} z^{2 \gamma}(z-y)^{2 \beta^{\prime}}}=\frac{V(\alpha, \beta)}{(x)^{2 \beta}(x-y)^{2 \gamma^{\prime}}(y)^{2 \alpha}} .
$$

Consider the dilatation operator $H:=(i / 2)(\hat{p} \hat{q}+\hat{q} \hat{p})=-H^{\dagger}$ which satisfies:

$$
H \hat{p}_{i}=\hat{p}_{i}(H-1), \quad H \hat{q}_{i}=\hat{q}_{i}(H+1),
$$


and generates the $\operatorname{sl}(2)$ algebra together with the elements $\hat{q}^{2}$ and $\hat{p}^{2}$ :

$$
\left[\hat{q}^{2}, \hat{p}^{2}\right]=4 H, \quad\left[H, \hat{q}^{2}\right]=2 \hat{q}^{2}, \quad\left[H, \hat{p}^{2}\right]=-2 \hat{p}^{2} .
$$

It is known that the special conformal transformation generators $K_{i}(4)$, the dilatation operator $H$ (11), the elements $\hat{p}_{i}$ and $M_{i j}=\hat{q}_{i} \hat{p}_{j}-\hat{q}_{j} \hat{p}_{i}$ generate the $D$ dimensional conformal algebra $s o(D, 2)$.

Below the following relations will be important:

$$
\begin{aligned}
{\left[\hat{q}^{2}, \hat{p}^{2(\alpha+1)}\right] } & =4(\alpha+1)(H+\alpha) \hat{p}^{2 \alpha}, \\
{\left[\hat{q}^{2(\alpha+1)}, \hat{p}^{2}\right] } & =4(\alpha+1)(H-\alpha) \hat{q}^{2 \alpha}, \\
H \hat{q}^{2 \alpha}=\hat{q}^{2 \alpha}(H+2 \alpha), & H \hat{p}^{2 \alpha}=\hat{p}^{2 \alpha}(H-2 \alpha) .
\end{aligned}
$$

These relations are easily deduced from the Heisenberg algebra (1). We also introduce the notion of degree for the operators which are homogeneous functions $\hat{\psi}\left(\hat{p}_{i}, \hat{q}_{j}\right)$ of the generators of the Heisenberg algebra. The additive number $\operatorname{deg}(\hat{\psi})$ is called the degree of the operator $\hat{\psi}$ if $[\hat{\psi}, H]=\operatorname{deg}(\hat{\psi}) \hat{\psi}$. In particular, we find $\operatorname{deg}\left(\hat{p}_{i}\right)=-\operatorname{deg}\left(\hat{q}_{j}\right)=1$ in view of $(11)$.

Using relations (13) and (15) one can deduce the algebraic identity

$$
\begin{gathered}
4(2 \gamma-\alpha-\beta) \hat{p}^{2 \alpha} \hat{q}^{2 \gamma} \hat{p}^{2 \beta}= \\
=\frac{1}{\alpha+1}\left[\hat{q}^{2}, \hat{p}^{2(\alpha+1)}\right] \hat{q}^{2 \gamma} \hat{p}^{2 \beta}-\frac{1}{\beta+1} \hat{p}^{2 \alpha} \hat{q}^{2 \gamma}\left[\hat{q}^{2}, \hat{p}^{2(\beta+1)}\right] .
\end{gathered}
$$

In the matrix form (5), (6) this identity looks like

$$
\begin{gathered}
\left(D-2 \alpha_{2}-\alpha_{1}-\alpha_{3}\right)\left\langle x\left|\alpha_{1}, \alpha_{2}, \alpha_{3}\right| y\right\rangle= \\
=\alpha_{1}\left[\left\langle x\left|\alpha_{1}^{+}, \alpha_{2}^{-}, \alpha_{3}\right| y\right\rangle-x^{2}\left\langle x\left|\alpha_{1}^{+}, \alpha_{2}, \alpha_{3}\right| y\right\rangle\right]+ \\
\left.+\alpha_{3}\left[\left\langle x\left|\alpha_{1}, \alpha_{2}^{-}, \alpha_{3}^{+}\right| y\right\rangle-y^{2}\left\langle x\left|\alpha_{1}, \alpha_{2}, \alpha_{3}^{+}\right| y\right\rangle\right)\right]
\end{gathered}
$$

where $\alpha_{i}^{ \pm}=\alpha_{i} \pm 1, \alpha=-\alpha_{1}^{\prime}, \gamma=-\alpha_{2}, \beta=-\alpha_{3}^{\prime}$ and we have introduced the concise notation for the "vertex" integral

$$
\left\langle x\left|\alpha_{1}, \alpha_{2}, \alpha_{3}\right| y\right\rangle=\int \frac{d^{D} z}{(x-z)^{2 \alpha_{1}} z^{2 \alpha_{2}}(z-y)^{2 \alpha_{3}}}=\frac{1}{a\left(\alpha_{1}^{\prime}\right) a\left(\alpha_{3}^{\prime}\right)}\left\langle x\left|\frac{1}{\hat{p}^{2 \alpha_{1}^{\prime}}} \frac{1}{\hat{q}^{2 \alpha_{2}}} \frac{1}{\hat{p}^{2 \alpha_{3}^{\prime}}}\right| y\right\rangle .
$$

Identity (17) is called the integration by parts (or "triangle") rule [9] and plays a very important role in almost all analytical calculations of multi-loop Feynman integrals.

The integration by parts rule (16) is a combination of two more fundamental relations. The first one is

$$
4(\alpha-\gamma) \hat{p}^{2 \alpha} \hat{q}^{2 \gamma}=\frac{1}{\gamma+1} \hat{p}^{2 \alpha}\left[\hat{q}^{2(\gamma+1)}, \hat{p}^{2}\right]-\frac{1}{\alpha+1}\left[\hat{q}^{2}, \hat{p}^{2(\alpha+1)}\right] \hat{q}^{2 \gamma},
$$

and the second one is obtained from (19) by the Hermitian conjugation (for real $\alpha$ and $\gamma$ ). These relations can also be deduced from (13) - (15).

Consider the sequence of products of the operators $\hat{p}^{2 \alpha_{2 k-1}}, \hat{q}^{2 \alpha_{2 k}}$

$$
\psi_{\bar{\alpha}}:=\psi\left(\ldots, \alpha_{i}, \ldots\right)=\cdots \hat{p}^{2 \alpha_{1}} \hat{q}^{2 \alpha_{2}} \hat{p}^{2 \alpha_{3}} \hat{q}^{2 \alpha_{4}} \ldots .
$$


Then the finite difference equation (19) and its conjugated version can be written in the form of equations $L_{i} \psi\left(\alpha_{i}\right)=0$ where the finite difference operators

$$
\begin{gathered}
L_{i}=4\left(\alpha_{i}-\alpha_{i+1}\right)+\nabla_{i}+\nabla_{i+1} \\
\left(\nabla_{i}=e^{\partial_{i}} \frac{1}{\alpha_{i}}\left[e^{\partial_{i-1}}-e^{\partial_{i+1}}\right], \quad \partial_{i}:=\frac{\partial}{\partial \alpha_{i}}\right),
\end{gathered}
$$

generate the algebra with the commutation relations: $\left[L_{i}, L_{i+k}\right]=0$ for $(k \geq 3)$ and

$$
\left[L_{i-1}-L_{i}, L_{i+1}-L_{i+2}\right]=0, \quad\left[L_{i}, L_{i+2}\right]=e^{\left(\partial_{i+1}+\partial_{i+2}\right)} \frac{1}{\alpha_{i+1} \alpha_{i+2}} L_{i+1} .
$$

This $W$ - type algebra has the central element $Z=-\frac{1}{4} \sum_{j}(-)^{j} L_{j}$ which is equal to the degree of the operator (20).

Another set of equations $\sigma_{i} \psi_{\bar{\alpha}}=\psi_{\bar{\alpha}}$ for the functions (20) follows from the star-triangle identity (9). The action of the operators $\sigma_{i}$ is

$$
\begin{gathered}
\sigma_{i} \psi\left(\ldots \alpha_{i}, \alpha_{i+1}, \alpha_{i+2}, \alpha_{i+3}, \ldots\right)= \\
=\psi\left(\ldots \alpha_{i}-\alpha_{i+1}+\alpha_{i+2}, \alpha_{i+2}, \alpha_{i+1}, \alpha_{i+1}-\alpha_{i+2}+\alpha_{i+3}, \ldots\right) .
\end{gathered}
$$

The operators (21) define the group $S_{\text {even }} \otimes S_{\text {odd }}$ which is a direct product of two symmetric groups generated by the sets of even $\left\{\sigma_{2 i}\right\}$ and odd $\left\{\sigma_{2 i+1}\right\}$ elements, respectively. One can show directly that the whole set of the relations $L_{i} \psi=0$ and $\sigma_{k} \psi=\psi$ is closed and combinations of these relations do not lead to new constraints on $\psi(20)$.

It is well known [17] that the dimensional regularization procedure requires the rule: $\int d^{D} x / x^{2 \alpha}=0$ for all $\alpha \neq D / 2$. A more precise statement [18] is

$$
\int d^{D} x \frac{1}{x^{2(D / 2+i \alpha)}}=\pi \Omega_{D} \delta(\alpha), \quad(\alpha \in R e)
$$

where $\Omega_{D}=\frac{2 \pi^{D / 2}}{\Gamma(D / 2)}$ is the area of the unit hypersphere in $R^{D}$.

In the framework of the dimensional regularization scheme we extend the definition of the integral in the left-hand side of (22) for arbitrary complex numbers $\alpha=|\alpha| e^{i \arg (\alpha)}$ as

$$
\int d^{D} x \frac{1}{x^{2(D / 2+\alpha)}}=\pi \Omega_{D} \delta(|\alpha|)
$$

where $\delta(|\alpha|)$ is the radial delta-function. It is clear that: $f(\alpha) \cdot \delta(|\alpha|)=f(0) \cdot \delta(|\alpha|)$ for analytic in $\alpha=0$ functions $f(\alpha)$.

The important consequence of the definition (23) is that now one can introduce the notion of the trace for the operators (20)

$$
\operatorname{Tr}\left(\psi_{\bar{\alpha}}\right)=\int d^{D} x\left\langle x\left|\psi_{\bar{\alpha}}\right| x\right\rangle .
$$

It follows from (23) that these traces are proportional to the delta-function:

$$
\operatorname{Tr}\left(\psi_{\bar{\alpha}}\right)=\pi \Omega_{D} c_{\alpha_{i}} \delta(|\beta|), \quad\left(c_{\alpha_{i}}=\int d \widetilde{x}\left\langle\widetilde{x}\left|\psi_{\bar{\alpha}}\right| \widetilde{x}\right\rangle\right)
$$

where $\beta=\frac{1}{2} \operatorname{deg}\left(\psi_{\bar{\alpha}}\right), \widetilde{x}_{i}=x_{i} / r$ is a unit vector in $R^{D}, d^{D} x=\Omega_{D} r^{D-1} d r d \widetilde{x}$ and $c_{\alpha_{i}}$ is a coefficient function which contains the whole information about the 
correlator $\left\langle x\left|\psi_{\bar{\alpha}}\right| x\right\rangle=c_{\alpha_{i}} x^{-2(D / 2+\beta)}$. The simple algebraic arguments which lead to the equation (25) are the following. Note that $e^{t\left(H+\frac{D}{2}\right)}|x\rangle=\left|e^{-t} x\right\rangle$ and for $r=e^{t}$ we obtain

$$
\left\langle x\left|\psi_{\bar{\alpha}}\right| x\right\rangle=\left\langle\widetilde{x}\left|e^{t\left(H-\frac{D}{2}\right)} \psi_{\bar{\alpha}} e^{-t\left(H+\frac{D}{2}\right)}\right| \widetilde{x}\right\rangle=e^{-t(2 \beta+D)}\left\langle\widetilde{x}\left|\psi_{\bar{\alpha}}\right| \widetilde{x}\right\rangle
$$

which is consistent with (25).

Remark 1. In the case $\operatorname{deg}\left(\psi_{\bar{\alpha}}\right)=0$, the operators $\psi_{\bar{\alpha}}(20)$ (which are cut off by the conditions $\alpha_{i}=0$ for $i<0$ and $i>2 k$ ) are expressed as a product of the commutative operators $H_{\alpha}$ and their inverse:

$$
\psi_{\bar{\alpha}}=H_{\alpha_{1}} H_{\alpha_{1}-\alpha_{2}}^{-1} H_{\alpha_{1}-\alpha_{2}+\alpha_{3}} \cdots H_{\alpha_{2 k}-\alpha_{2 k-1}}^{-1} H_{\alpha_{2 k}}, \quad\left(\operatorname{deg}\left(H_{\alpha}\right)=0\right) .
$$

In this case the problem of calculation of the trace (24) reduces to the spectral problem for the commutative operators $H_{\alpha}$.

Remark 2. The star-triangle identity (9) can be generalized to

$$
\begin{aligned}
& \sum_{i_{1} \ldots i_{n}}\left(\hat{p}^{2 \alpha} h_{i_{1} \ldots i_{k}}^{(1)}(\hat{p})\right) \hat{q}^{2(\alpha+\beta)} h_{i_{1} \ldots i_{n}}^{(2)}(\hat{q})\left(\hat{p}^{2 \beta} h_{i_{k+1} \ldots i_{n}}^{(3)}(\hat{p})\right)= \\
& =\sum_{i_{1} \ldots i_{n}} \hat{q}^{2 \beta} h_{i_{k+1} \ldots i_{n}}^{(3)}(\hat{q})\left(\hat{p}^{2(\alpha+\beta)} h_{i_{1} \ldots i_{n}}^{(2)}(\hat{p})\right) \hat{q}^{2 \alpha} h_{i_{1} \ldots i_{k}}^{(1)}(\hat{q}),
\end{aligned}
$$

where $h_{i_{1} \ldots i_{k}}^{(a)}().(a=1,2,3)$ are any tensors being $k$ th-order homogeneous polynomials in $\hat{q}_{i}$ or $\hat{p}_{i}\left(\operatorname{deg}\left(h_{i_{1} \ldots i_{k}}(\hat{p})\right)=-\operatorname{deg}\left(h_{i_{1} \ldots i_{k}}(\hat{q})\right)=k\right)$.

Remark 3. The following relation holds:

$$
\frac{1}{(\hat{q}-y)^{2 \beta}} \frac{a(\alpha)}{\hat{p}^{2 \alpha^{\prime}}}|x\rangle=\frac{1}{(\hat{q}-x)^{2 \alpha}} \frac{a(\beta)}{\hat{p}^{2 \beta^{\prime}}}|y\rangle .
$$

Indeed, the contraction of both sides of (28) with an arbitrary state $\langle z|$ and relation (5) give the identity. One deduces from (28) that the vector $a(\alpha)(\hat{q}-x)^{2 \alpha} \hat{p}^{-2 \alpha^{\prime}}|x\rangle$ is independent of the parameters $\alpha$ and $x_{i}$ and, thus, should be canceled by the operators $\hat{p}_{i}$.

Remark 4. The dual analog $\bar{R}$ of the inversion operator (4) can be defined as

$$
\begin{gathered}
\bar{R} \hat{p}_{i} \bar{R}=\hat{p}_{i} / \hat{p}^{2}, \quad \bar{R} \hat{q}_{i} \bar{R}=\hat{p}^{2} \hat{q}_{i}-2 \hat{p}_{i}(\hat{p} \hat{q}), \\
\bar{R} \hat{q}^{2 \beta} \bar{R}=\hat{p}^{2\left(\beta+\frac{D}{2}\right)} \hat{q}^{2 \beta} \hat{p}^{2\left(\beta-\frac{D}{2}\right)} .
\end{gathered}
$$

where $\bar{R}^{2}=1, \bar{R}^{\dagger}=\bar{R} \hat{p}^{2 D}$ and $\langle k| \bar{R}=\left\langle\frac{1}{k}\right|$.

Remark 5. Certain algebraic identities considered above (e.g. eqs. (13) and (14)) are related to each other by the obvious $Z_{2}$ symmetry $\hat{p}^{2} \leftrightarrow \hat{q}^{2}, H \leftrightarrow-H$ which is the well known automorphism of the $s l(2)$ algebra (12). 


\section{The Diagrams}

The Feynman diagrams which will be considered in this paper are graphs with vertices connected by lines labeled by numbers (indices). With each vertex we associate the point in the D-dimensional space $R^{D}$ while the lines of the graph (with index $\alpha$ ) are associated with the propagator

$$
x+\frac{\alpha}{x}=1 /(x-y)^{2 \alpha}
$$

The boldface vertices $\bullet$ denote that the corresponding points are integrated over $R^{D}$. These diagrams are called the Feynman diagrams in the configuration space.

The figures and operator form of integral expressions for the 3-point, 2-point diagrams and the tetrahedron vacuum diagram are

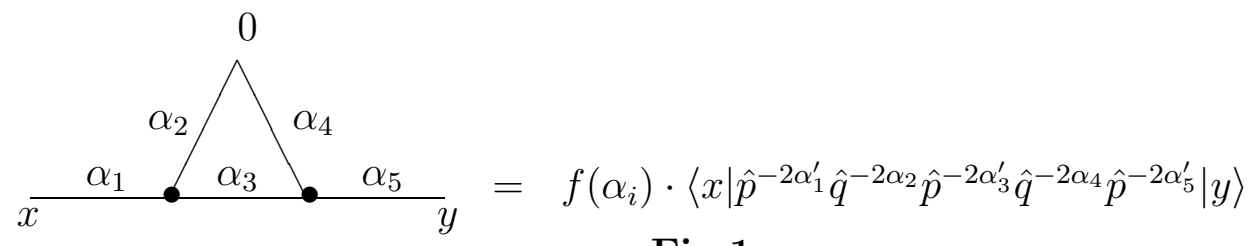

Fig.1

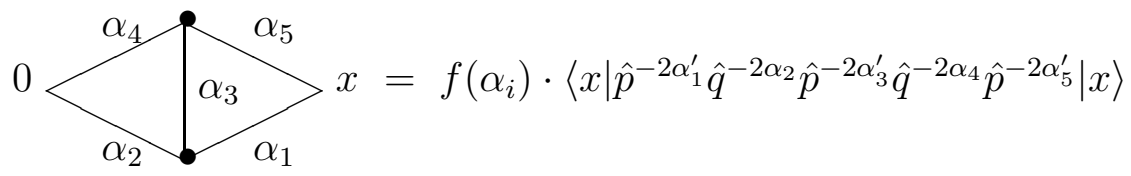

Fig.2

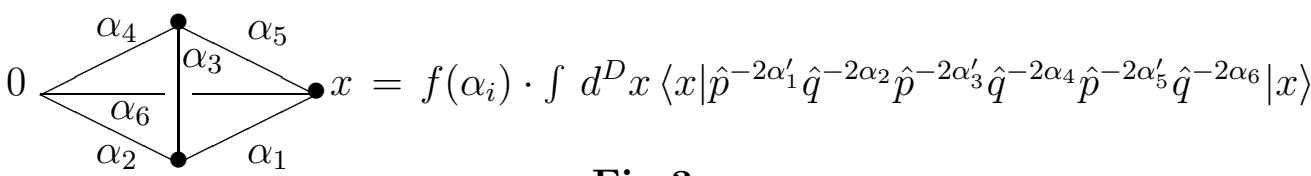

Fig.3

where the factor $f\left(\alpha_{i}\right)$ is equal to $(2 \pi)^{3 D} a\left(\alpha_{1}\right) a\left(\alpha_{3}\right) a\left(\alpha_{5}\right)$.

The two-loop propagator-type diagram on Fig. 2 (which is called in [19] the master two-loop diagram) is obtained from the diagram in Fig. 1 in the case $x=y$. The expression for the vacuum "tetrahedron" diagram in Fig.3 is the result of the integration of the expression for the master two-loop diagram with the additional propagator $1 / x^{2 \alpha_{6}}$. This expression is represented in the form of the trace $(24)$

$$
f\left(\alpha_{i}\right) \cdot \operatorname{Tr}\left(\hat{p}^{-2 \alpha_{1}^{\prime}} \hat{q}^{-2 \alpha_{2}} \hat{p}^{-2 \alpha_{3}^{\prime}} \hat{q}^{-2 \alpha_{4}} \hat{p}^{-2 \alpha_{5}^{\prime}} \hat{q}^{-2 \alpha_{6}}\right) \text {. }
$$

The tetrahedral symmetry [18] of this diagram becomes evident if we impose, in addition to the cyclic symmetry of the trace $\left(s_{1}^{3}=1\right)$ :

$$
\begin{aligned}
& s_{1}: \operatorname{Tr}\left(\hat{p}^{-2 \alpha_{1}^{\prime}} \hat{q}^{-2 \alpha_{2}} \hat{p}^{-2 \alpha_{3}^{\prime}} \hat{q}^{-2 \alpha_{4}} \hat{p}^{-2 \alpha_{5}^{\prime}} \hat{q}^{-2 \alpha_{6}}\right)= \\
& \operatorname{Tr}\left(\hat{p}^{-2 \alpha_{3}^{\prime}} \hat{q}^{-2 \alpha_{4}} \hat{p}^{-2 \alpha_{5}^{\prime}} \hat{q}^{-2 \alpha_{6}} \hat{p}^{-2 \alpha_{1}^{\prime}} \hat{q}^{-2 \alpha_{2}}\right),
\end{aligned}
$$

the identity (the reflection of the diagram in Fig.3 with respect to the vertical line; 
this reflection is equivalent to the permutation of the vertices $x$ and $0 ; s_{2}^{2}=1$ ):

$$
\begin{aligned}
& s_{2}: \operatorname{Tr}\left(\hat{p}^{-2 \alpha_{1}^{\prime}} \hat{q}^{-2 \alpha_{2}} \hat{p}^{-2 \alpha_{3}^{\prime}} \hat{q}^{-2 \alpha_{4}} \hat{p}^{-2 \alpha_{5}^{\prime}} \hat{q}^{-2 \alpha_{6}}\right)= \\
& =\frac{a\left(\alpha_{2}\right) a\left(\alpha_{4}\right)}{a\left(\alpha_{1}\right) a\left(\alpha_{5}\right)} \operatorname{Tr}\left(\hat{p}^{-2 \alpha_{2}^{\prime}} \hat{q}^{-2 \alpha_{1}} \hat{p}^{-2 \alpha_{3}^{\prime}} \hat{q}^{-2 \alpha_{5}} \hat{p}^{-2 \alpha_{4}^{\prime}} \hat{q}^{-2 \alpha_{6}}\right),
\end{aligned}
$$

The last identity is deduced from (28). With the help of the transformations $s_{1}$ and $s_{2}$ one can permute every two vertices of the tetrahedron on Fig.3. Thus, the elements $s_{1}, s_{2}$ generate the permutation group $S_{4}$ (e.g. one can check that the element $\left(s_{1} s_{2}\right)$ generates the 4th-order symmetry $\left.\left(s_{1} s_{2}\right)^{4}=1\right)$.

Following the paper [18] we add to (31) and (32) the symmetry (cf. with (21)) deduced from the star-triangle equation $\left(s_{3}^{2}=1\right)$ :

$$
\begin{aligned}
& s_{3}: \operatorname{Tr}\left(\hat{p}^{-2 \alpha_{1}^{\prime}} \hat{q}^{-2 \alpha_{2}} \hat{p}^{-2 \alpha_{3}^{\prime}} \hat{q}^{-2 \alpha_{4}} \hat{p}^{-2 \alpha_{5}^{\prime}} \hat{q}^{-2 \alpha_{6}}\right)= \\
& =\operatorname{Tr}\left(\hat{p}^{-2\left(\alpha_{1}^{\prime}-\alpha_{2}+\alpha_{3}^{\prime}\right)} \hat{q}^{-2 \alpha_{3}^{\prime}} \hat{p}^{-2 \alpha_{2}} \hat{q}^{-2\left(\alpha_{2}-\alpha_{3}^{\prime}+\alpha_{4}\right)} \hat{p}^{-2 \alpha_{5}^{\prime}} \hat{q}^{-2 \alpha_{6}}\right),
\end{aligned}
$$

The symmetries $s_{1}, s_{2}, s_{3}(31)-(33)$ can be realized as linear transformations of the indices $\alpha_{i}$ and, as it was shown in [20], they generate the 1440-dimensional finite group $S_{6} \otimes Z_{2}$, where $S_{6}$ is a group of permutations of 6 objects and $Z_{2}$ is a 2-fold cyclic group. The most general analytical result for the master two-loop diagram on Fig. 2 has been achieved in [21] and [22]. According to this result, the trace (30) for $\alpha_{4}=\alpha_{2}=1$ can be expressed in terms of ${ }_{3} F_{2}$ hypergeometric series.

At the end of this section we stress that to write down more complicated diagrams in the operator form, we need to extend the Heisenberg algebra (1) to the multiparticle case $\left[\hat{q}_{k}^{(a)}, \hat{p}_{j}^{(b)}\right]=i \delta_{k j} \delta^{a b}$, where $a, b=1,2, \ldots$ are the numbers of particles.

\section{Applications.}

In this Section we demonstrate how our algebraic methods work using the example of the $L$-loop ladder ( $L$-boxes) diagrams. We start with dimensionally and analytically regularized massless integrals

$$
D_{L}\left(p_{0}, p_{L+1}, p ; \alpha, \beta, \gamma\right)=\left[\prod_{k=1}^{L} \int \frac{d^{D} p_{k}}{p_{k}^{2 \alpha_{k}}\left(p_{k}-p\right)^{2 \beta_{k}}}\right] \prod_{m=0}^{L} \frac{1}{\left(p_{m+1}-p_{m}\right)^{2 \gamma_{m}}}
$$

which correspond to the diagram $\left(x=p_{0}, y=p_{L+1}, z=p\right)$ :

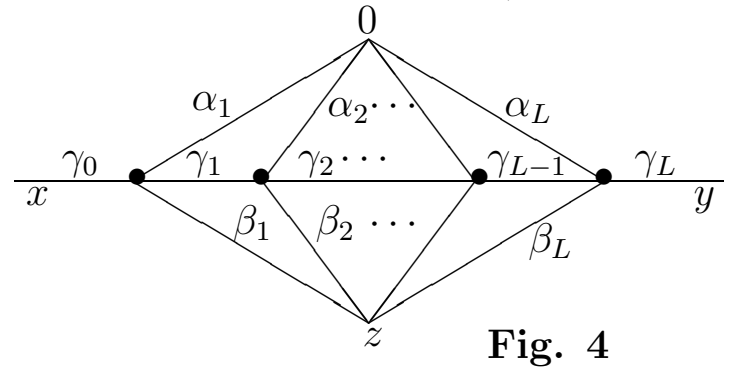

The dual to this diagram is the ladder $L$-loop diagram for the massless $\phi^{3}$ theory (the loops in Fig. 5 correspond to the boldface vertices in Fig.4): 


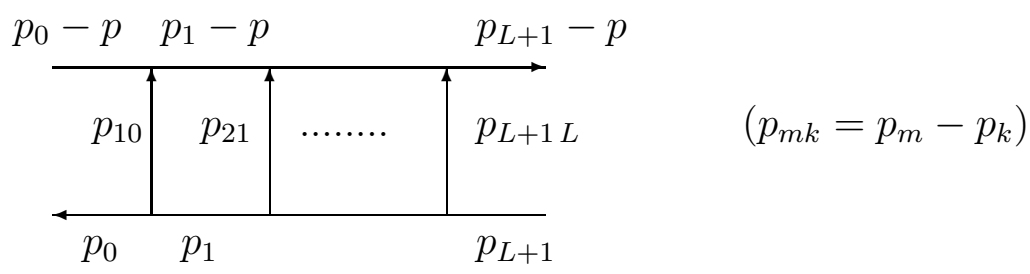

Fig. 5

This "momentum space" diagram represents the same integral (34), but in another graphical form. Using (3) and (5) we obtain for (34) the following representation:

$$
D_{L}(x, y, z ; \alpha, \beta, \gamma)=\left(\prod_{k=0}^{L} \frac{1}{a\left(\gamma_{k}^{\prime}\right)}\right)\left\langle x\left|\frac{1}{\hat{p}^{2 \gamma_{0}^{\prime}}}\left(\prod_{k=1}^{L} \frac{1}{\hat{q}^{2 \alpha_{k}}} \frac{1}{(\hat{q}-z)^{2 \beta_{k}}} \frac{1}{\hat{p}^{2 \gamma_{k}^{\prime}}}\right)\right| y\right\rangle .
$$

Now we simplify the problem by fixing indices of the propagators as $\alpha_{k}=\alpha$, $\beta_{k}=\beta, \gamma_{k}=\gamma$, and consider the generating function

$$
\begin{gathered}
D_{g}(x, y, z ; \alpha, \beta, \gamma):=a\left(\gamma^{\prime}\right) \sum_{L=0}^{\infty} g^{L} D_{L}(x, y, z ; \alpha, \beta, \gamma)= \\
=\left\langle x\left|\left(\hat{p}^{2 \gamma^{\prime}}-\frac{\bar{g}}{\hat{q}^{2 \alpha}(\hat{q}-z)^{2 \beta}}\right)^{-1}\right| y\right\rangle
\end{gathered}
$$

where $\bar{g}=g / a\left(\gamma^{\prime}\right)$ is the renormalized coupling constant. The right-hand side of (36) is the Green function for the two-center operator

$$
\mathcal{H}=\hat{p}^{2 \gamma^{\prime}}-\frac{\bar{g}}{\hat{q}^{2 \alpha}(\hat{q}-z)^{2 \beta}} .
$$

This Green function has the following symmetries under the linear and inverse transformations of the coordinates $(x, y, z)$ :

$$
\begin{gathered}
D_{g}(x, y, z ; \alpha, \beta, \gamma)=D_{g}(y, x, z ; \alpha, \beta, \gamma), \\
D_{g}(x-z, y-z,-z ; \beta, \alpha, \gamma)=D_{g}(x, y, z ; \alpha, \beta, \gamma), \\
D_{g z^{-2 \beta}}\left(\frac{1}{x}, \frac{1}{y}, \frac{1}{z} ; \widetilde{\alpha}, \beta, \gamma\right)=x^{2 \gamma} y^{2 \gamma} D_{g}(x, y, z ; \alpha, \beta, \gamma) .
\end{gathered}
$$

Here $1 / x:=\left\{x_{i} / x^{2}\right\}$ and

$$
\widetilde{\alpha}=2 \gamma^{\prime}-\alpha-\beta=D-(\alpha+\beta+2 \gamma) .
$$

The proof of (37) and (38) is evident. The symmetry (39) follows from the chain of equalities

$$
\begin{gathered}
\left\langle x\left|R^{2}\left(\hat{p}^{2 \gamma^{\prime}}-\frac{\bar{g}}{\hat{q}^{2 \alpha}(\hat{q}-z)^{2 \beta}}\right)^{-1}\right| y\right\rangle= \\
=\left\langle x\left|R\left(\hat{q}^{2\left(\gamma^{\prime}+D / 2\right)} \hat{p}^{2 \gamma^{\prime}} \hat{q}^{2\left(\gamma^{\prime}-D / 2\right)}-\frac{\bar{g} z^{-2 \beta}}{\hat{q}^{-2(\alpha+\beta)}\left(\hat{q}-\frac{1}{z}\right)^{2 \beta}}\right)^{-1} R\right| y\right\rangle= \\
=x^{-2 \gamma} y^{-2 \gamma}\left\langle\frac{1}{x}\left|\left(\hat{p}^{2 \gamma^{\prime}}-\frac{\bar{g} z^{-2 \beta}}{\hat{q}^{2 \widetilde{\alpha}}\left(\hat{q}-\frac{1}{z}\right)^{2 \beta}}\right)^{-1}\right| \frac{1}{y}\right\rangle
\end{gathered}
$$


where equations (4) and (8) where applied.

Using identities (38) and (39) one can change the indices of the propagators in (34). In the case $\widetilde{\alpha}=0$ (see (40)) the combination of the symmetries (38) and (39) gives

$$
D_{g}(x, y, z ; \alpha, \beta, \gamma)=x^{-2 \gamma} y^{-2 \gamma} G_{\gamma^{\prime}, \beta}(u, v)
$$

where

$$
G_{\gamma^{\prime}, \beta}(u, v)=\left\langle u\left|\left(\hat{p}^{2 \gamma^{\prime}}-\frac{g_{z}}{\hat{q}^{2 \beta}}\right)^{-1}\right| v\right\rangle,
$$

and

$$
g_{z}=\bar{g} z^{-2 \beta}=\frac{g}{a\left(\gamma^{\prime}\right) z^{2 \beta}}, \quad u_{i}=\frac{x_{i}}{x^{2}}-\frac{z_{i}}{z^{2}}, \quad v_{i}=\frac{y_{i}}{y^{2}}-\frac{z_{i}}{z^{2}} .
$$

The function $G_{\gamma^{\prime}, \beta}(x, y)$ is obviously related to the function (36) (if we put there $\beta=0, \bar{g}=g_{z}$ ), and for the case $g_{z}=$ const from (37), (39) we obtain the symmetry

$$
G_{\gamma, \beta}(u, v)=G_{\gamma, \beta}(v, u)=\left(u^{2} v^{2}\right)^{\left(\gamma-\frac{D}{2}\right)} G_{\gamma, 2 \gamma-\beta}\left(\frac{1}{u}, \frac{1}{v}\right),
$$

(note that here we have made the redefinition: $\gamma^{\prime} \rightarrow \gamma$ ).

Thus, the problem of analytical evaluation of the integrals (34) for the ladder diagrams with the special choice of the line indices $(\alpha+\beta+2 \gamma=D)$ is reduced to the problem of calculation of the Green function for the Hamiltonian

$$
\mathcal{H}=\hat{p}^{2 \gamma^{\prime}}-\frac{g_{z}}{\hat{q}^{2 \beta}} .
$$

which describes (for $\gamma^{\prime}=1$ ) the propagation of the scalar particle in the external field of one source fixed in the origin. Now the most interesting cases are:

1.) $\gamma^{\prime}=\beta=1,(\alpha=1)$ which corresponds to the $D$-dimensional conformal mechanics;

2.) $\gamma^{\prime}=1, \beta=D / 2-1,\left(\alpha=3-\frac{D}{2}\right)$ which is related to the standard $D$-dimensional problem of the dynamics of a charged particle moving in the field of the pointlike source. For $D=4$ both the cases are equivalent to each other and the Green function (43) defines the generating function for the ladder diagrams in the $\phi^{3}(D=4)$ field theory.

Here we calculate the Green function (43) and, therefore, the integral (34) for general value of $D$ and for the choice of the indices $\gamma=D / 2-1, \alpha=\beta=1$ ( $D$-dimensional conformal mechanics) with the help of the operator approach. Our method is based on the identity:

$$
\frac{1}{\hat{p}^{2}-g / \hat{q}^{2}}=\sum_{L=0}^{\infty}\left(-\frac{g}{4}\right)^{L}\left[\hat{q}^{2 \alpha} \frac{(H-1)}{(H-1+\alpha)^{L+1}} \frac{1}{\hat{p}^{2}} \hat{q}^{-2 \alpha}\right]_{\alpha^{L}}
$$

where we denote $[\ldots]_{\alpha^{L}}=\frac{1}{L !}\left(\partial_{\alpha}^{L}[\ldots]\right)_{\alpha=0}$. Identity (45) can be proved immediately if one acts on both the sides by $\mathcal{H}=\hat{p}^{2}-g / \hat{q}^{2}$ and uses relations (13)-(15) and $\left[(h-2 \alpha)(h-\alpha)^{-L-1}\right]_{\alpha^{L}}=\delta_{L, 0}, \forall h \neq 0$. Taking into account the integral representation for the rational function of $H$ in (45)

$$
\frac{(H-1)}{(H-1+\alpha)^{L+1}}=\frac{(-1)^{L+1}}{L !} \int_{0}^{\infty} d t t^{L} e^{t \alpha} \partial_{t}\left(e^{t(H-1)}\right)
$$


the Green function $G_{1,1}(u, v)(43)$ is written in the form

$$
\left\langle u\left|\frac{1}{\left(\hat{p}^{2}-g_{z} / \hat{q}^{2}\right)}\right| v\right\rangle=\sum_{L=0}^{\infty} \frac{1}{L !}\left(\frac{g_{z}}{4}\right)^{L} \Phi_{L}(u, v)
$$

where for the functions $\Phi_{L}(u, v)$ we obtain the integral representation

$$
\begin{gathered}
\Phi_{L}(u, v)=-\int_{0}^{\infty} d t t^{L}\left[\left\langle u\left|\left(\hat{q}^{2} e^{t}\right)^{\alpha} \partial_{t}\left(e^{t(H-1)}\right) \frac{1}{\hat{p}^{2}} \hat{q}^{-2 \alpha}\right| v\right\rangle\right]_{\alpha^{L}}= \\
=-a(1) \int_{0}^{\infty} d t t^{L}\left[\left(\frac{u^{2}}{v^{2}}\right)^{\alpha} e^{t \alpha}\right]_{\alpha^{L}} \partial_{t}\left(\frac{e^{-t}}{\left(u-e^{-t} v\right)^{2}}\right)^{\left(\frac{D}{2}-1\right)}
\end{gathered}
$$

Note that the first function is: $\Phi_{0}=a(1)(u-v)^{2\left(1-\frac{D}{2}\right)}$ (as it also follows from (46)) and in view of (44) all $\Phi_{L}$ possess the symmetry

$$
\Phi_{L}(u, v)=\Phi_{L}(v, u)=\left(u^{2} v^{2}\right)^{\left(1-\frac{D}{2}\right)} \Phi_{L}\left(\frac{1}{u}, \frac{1}{v}\right) .
$$

The integral (47) for $D=4$ reproduces the results of [23] and [24]. For applications it is worth having the general expression (47) for $D=4-2 \epsilon$ and expand $\Phi_{L}(u, v)$ over small $\epsilon$ :

$$
\Phi_{L}(u, v)=\frac{\Gamma(1-\epsilon)}{4 \pi^{2-\epsilon} u^{2(1-\epsilon)}} \sum_{l=0}^{\infty} \frac{\epsilon^{l}}{l !} \Phi_{L}^{(l)}\left(z_{1}, z_{2}\right) .
$$

Here the dimensionless parameters $z_{1}, z_{2}$ are defined by the equations: $z_{1}+z_{2}=$ $2(u v) / u^{2}$ and $z_{1} z_{2}=v^{2} / u^{2}$. The coefficient functions $\Phi_{L}^{(l)}$ in the expansion (48) can be represented in the following form:

$$
\Phi_{L}^{(l)}\left(z_{1}, z_{2}\right)=\sum_{f=0}^{L} \frac{\left(-\ln \left(z_{1} z_{2}\right)\right)^{f}(2 L-f)}{f !(L-f) !} \sum_{m=0}^{l}(-)^{m} C_{l}^{m} \mathbf{Z}_{m}\left(z_{1}, z_{2} ; 2 L+l-f\right)
$$

where $C_{l}^{m}=\frac{l !}{m !(l-m) !}$ and we denote $(k>m)$ :

$$
\mathbf{Z}_{m}\left(z_{1}, z_{2} ; k\right)=\frac{\Gamma(k-m)}{\left(z_{1}-z_{2}\right)} \sum_{\left\{n_{i}\right\}=1}^{\infty} \frac{\left(z_{1}^{n_{0}}-z_{2}^{n_{0}}\right)}{\left(\sum_{0}^{m} n_{i}\right)^{k-m}}\left(\prod_{i=1}^{m} \frac{z_{1}^{n_{i}}+z_{2}^{n_{i}}}{n_{i}}\right)
$$

For example, we have

$$
\begin{gathered}
\mathbf{Z}_{0}\left(z_{1}, z_{2} ; k\right)=\frac{\Gamma(k)}{z_{1}-z_{2}}\left(\operatorname{Li}_{k}\left(z_{1}\right)-\operatorname{Li}_{k}\left(z_{2}\right)\right) \\
\mathbf{Z}_{1}\left(z_{1}, z_{2} ; k\right)=\frac{\Gamma(k-1)}{z_{1}-z_{2}}\left(\operatorname{Li}_{k-1,1}\left(z_{1}, 1\right)+\operatorname{Li}_{k-1,1}\left(z_{1}, \frac{z_{2}}{z_{1}}\right)-\left(z_{1} \leftrightarrow z_{2}\right)\right),
\end{gathered}
$$

and in general the functions $\mathbf{Z}_{m}\left(z_{1}, z_{2} ; k\right)$ are linear combinations of multiple polylogarithms

$$
\operatorname{Li}_{k-m, m_{1}, \ldots, m_{r}}\left(w_{0}, w_{1}, \ldots, w_{r}\right)=\sum_{n_{0}>n_{1}>\ldots>n_{r}>0} \frac{w_{0}^{n_{0}} w_{1}^{n_{1}} \cdots w_{r}^{n_{r}}}{n_{0}^{k-m} n_{1}^{m_{1}} \ldots n_{r}^{m_{r}}}
$$


with $\sum m_{i}=m$ and arguments $w_{i}$ belong to the set $\left\{1, z_{1}, z_{2}, \frac{z_{1}}{z_{2}}, \frac{z_{2}}{z_{1}}, \frac{z_{1}^{2}}{z_{2}^{2}} \ldots\right\}$. The first coefficient function in (49) (related to the integral (47) for $D=4$ )

$$
\Phi_{L}^{(0)}\left(z_{1}, z_{2}\right)=\frac{1}{z_{1}-z_{2}} \sum_{f=0}^{L} \frac{(-)^{f}(2 L-f) !}{f !(L-f) !} \ln ^{f}\left(z_{1} z_{2}\right)\left[\operatorname{Li}_{2 L-f}\left(z_{1}\right)-\operatorname{Li}_{2 L-f}\left(z_{2}\right)\right]
$$

has firstly been evaluated in [23]. The next coefficient is

$$
\Phi_{L}^{(1)}\left(z_{1}, z_{2}\right)=\sum_{n=L}^{2 L} \frac{n\left(-\ln \left(z_{1} z_{2}\right)\right)^{2 L-n}}{(2 L-n) !(n-L) !}\left(\mathbf{Z}_{0}\left(z_{1}, z_{2} ; n+1\right)-\mathbf{Z}_{1}\left(z_{1}, z_{2} ; n+1\right)\right),
$$

We also present the result for the integral (47) in the case $u=v(x=y)$ and arbitrary $D<2(L+1)$ which is needed for evaluation of the propagator-type ladder diagrams:

$$
u^{2\left(\frac{D}{2}-1\right)} \Phi_{L}(u, u)=\frac{a(1)(2 L-1) ! !}{\Gamma(D-2)} \sum_{k=0}^{\infty} \frac{\Gamma(k+D-2)}{k !(k+D / 2-1)^{2 L}} .
$$

For $D=4$ we immediately obtain $u^{2} \Phi_{L}(u, u) \sim \zeta(2 L-1)$.

Remark 1. Relation (14) can be rewritten in the form

$$
\frac{1}{\hat{p}^{2}} \hat{q}^{2(\alpha-1)} \frac{1}{\hat{p}^{2}}=\frac{-1}{4 \alpha(H-\alpha-1)}\left[\hat{q}^{2 \alpha}, \frac{1}{\hat{p}^{2}}\right]=\frac{1}{4 \alpha} \int_{0}^{\infty} d t e^{t(H-\alpha-1)}\left[\hat{q}^{2 \alpha}, \frac{1}{\hat{p}^{2}}\right] .
$$

In the matrix representation (5), (6) this identity gives the analytical expression for the vertex integral (18) for the special choice of the indices (one index is arbitrary)

$$
\left\langle x\left|\frac{D}{2}-1,1-\alpha, \frac{D}{2}-1\right| y\right\rangle=\frac{1}{4 \alpha a(1)} \int_{0}^{\infty} d t e^{t\left(\frac{D}{2}-1\right)} \frac{\left(x^{2} e^{t}\right)^{\alpha}-\left(y^{2} e^{-t}\right)^{\alpha}}{\left(e^{t} x-y\right)^{2\left(\frac{D}{2}-1\right)}}
$$

It reproduces the results presented in [26], [27] since the indices of the lines in the left-hand side of (51) are changed by means of the star-triangle symmetries (21).

Remark 2. The one- and two-loop ladder integrals (34) for the case $D=4$ and $\alpha_{k}=\beta_{k}=\gamma_{k}=1$ (which are given by the functions $\Phi_{1}^{(0)}$ and $\Phi_{2}^{(0)}(50)$ ) have been found in [25]. This result was generalized in [23] and [24] where (50) for all $L$ and the generating function (46) (for the case $D=4$ and $\alpha_{k}=\beta_{k}=\gamma_{k}=1$ ) has been calculated. The analytical results for 1,2,3-loop (boxes) integrals (34), general value of $D$ and special indices of the lines $\alpha_{k}, \beta_{k}, \gamma_{k}$ can be found in [4], [27], [28], [29], [30] (see also references therein).

Remark 3. In the case of the conformal indices: $\alpha_{k}+\beta_{k}+\gamma_{k}+\gamma_{k-1}=D(\forall k)$ the inversion method (41) reduces the 4-point functions (34), (35) (represented on Fig. 4) to the 3-point functions. Moreover, the inversion method (41) gives the possibility to obtain the duality transformation for the $D$-dimensional Green function

$$
\left\langle x\left|\frac{1}{\hat{p}^{2 \alpha}+g\left(\hat{q}^{2}\right)^{-2 \alpha}+E}\right| y\right\rangle=\left(x^{2} y^{2}\right)^{\left(\alpha-\frac{D}{2}\right)}\left\langle\frac{1}{x}\left|\frac{1}{\hat{p}^{2 \alpha}+g+E\left(\hat{q}^{2}\right)^{-2 \alpha}}\right| \frac{1}{y}\right\rangle
$$


which relates (for the fixed parameters $\alpha=1, E=0$ ) the $D$-dimensional Green function for the Hamiltonian $\mathcal{H}=\hat{p}^{2}+g\left(\hat{q}^{2}\right)^{-2}$ to the propagator of a massive $\left(m^{2}=\right.$ $g \mu^{2}$ ) free particle (the dimension parameter $\mu$ can be restored by the renormalization $\hat{p} \rightarrow \hat{p} / \mu$ and $\hat{q} \rightarrow \mu \hat{q})$.

The inversion method (41) in the momentum representation (with the help of the operator $\bar{R}(29))$ gives the duality transformation for the Green function

$$
\left(k^{2} \tilde{k}^{2}\right)^{\frac{(\alpha+D)}{2}}\left\langle k\left|\left(\hat{p}^{2 \alpha}+\frac{g}{\left(\hat{q}^{2}\right)^{\frac{\alpha}{2}}}+E\right)^{-1}\right| \tilde{k}\right\rangle=\left\langle\frac{1}{k}\left|\left(1+\frac{g}{\left(\hat{q}^{2}\right)^{\frac{\alpha}{2}}}+E \hat{p}^{2 \alpha}\right)^{-1}\right| \frac{1}{\tilde{k}}\right\rangle,
$$

where $g$ and $E$ are dimensionless parameters. For $\alpha=1, D=3$ we have deduced the duality transformation for the Green function of the Coulomb problem.

\section{Conclusion}

In this paper the algebraic approach to analytical calculations of the integrals for multi-loop Feynman diagrams is developed. Possible modifications and applications of the approach are the following. First of all we plan to extend the method to the case of massive propagators. It is rather trivial to generalize the integration by parts identities (16), (19) to the massive case. The massive generalization of the star-triangle identities (9), (27) is unknown (see, however, Sect. 3.2 in the paper $[3])$.

The algebraic formulation of the Gegenbauer polynomial technique [31], [22] is also possible and based on the identity

$$
\frac{1}{a\left(\alpha^{\prime}\right)} \frac{1}{\hat{p}^{2 \alpha^{\prime}}}=\sum_{n=0}^{\infty} \hat{q}^{n}\left[\int_{w^{2}>z^{2}} d^{D} z d^{D} w|z\rangle C_{n}^{\alpha}(\tilde{z} \tilde{w})\langle w|+(w \leftrightarrow z)\right] \hat{q}^{-n-2 \alpha},
$$

where $\tilde{z} \tilde{w}=(w z) /\left(w^{2} z^{2}\right)^{\frac{1}{2}}$ and $C_{n}^{\alpha}(t)$ are Gegenbauer polynomials. It is tempting to apply (52) for the evaluation of the two-loop master diagram on Fig. 2.

Finally we stress that our methods resemble the methods used in the theory of the quantum integrable systems. For example, the star-triangle identity (10) is a kind of the Yang-Baxter equation [13], [14] and can be used for the $R$-matrix formulation [32] of the integrable noncompact Heisenberg spin chain proposed by Lipatov [33] for describing the high-energy scattering of hadrons in QCD. So we hope that the algebraic approach developed in this paper will be helpful in investigations of the Lipatov's model.

Acknowledgments. I would like to thank G. Arutyunov, D. Broadhurst, A.I. Davydychev, H. Gangl, D.I. Kazakov, A.V. Kotikov, L.N. Lipatov, O.V. Ogievetsky, V.A. Smirnov, F.V. Tkachov, A.A. Vladimirov and A.B. Zamolodchikov for interesting discussions and comments. I also thank the Max-Planck-Institut für Mathematik in Bonn, where in the beginning of 2002 the considerable part of this work was done, for their kind hospitality and support. 


\section{References}

[1] D. Kreimer, Phys. Rept. 363 (2002) 387, hep-th/0010059; D. Kreimer, Knots and Feynman Diagrams, Cambridge Univ. Press (2000).

[2] D.J. Broadhurst, Eur. Phys. J. C 8 (1999) 311, hep-th/9803091.

[3] A.I. Davydychev and R. Delbourgo, J. Math. Phys. 39 (1998) 4299, hepth/9709216.

[4] V.A. Smirnov, Evaluating double and triple (?) boxes, hep-ph/0209295; Phys. Lett. B 547 (2002) 239, hep-ph/0209193; Phys. Lett. B 500 (2001) 330, hepph/0011056; Phys. Lett. B 460 (1999) 397, hep-ph/9905323.

[5] S. Moch, P. Uwer and S. Weinzierl, J. Math. Phys. 43 (2002) 3363, hep$\mathrm{ph} / 0110083$.

[6] A.I. Davydychev and M.Yu. Kalmykov, Nucl. Phys. B 605 (2001) 266; hep-th/0012189.

[7] D. Zagier, Values of Zeta-functions and their applications, In First Europian Congress of Mathematics, Vol. II, Birkhauser, Boston, 1994, 497.

[8] A.B. Goncharov, Multiple polylogarithms and mixed Tate motives, math.AG/0103059.

[9] F.V. Tkachov, Phys. Lett. B 100 (1981) 65; K.G. Chetyrkin and F.V. Tkachov, Nucl. Phys. B 192 (1981) 159.

[10] D.I. Kazakov, Phys. Lett. B 133 (1983) 406; Theor. Math. Phys. 62, 84 (1985).

[11] A. N. Vasilev, Y. M. Pismak and Y. R. Khonkonen, Theor. Math. Phys. 47 (1981) 465.

[12] E.S. Fradkin and M.Ya. Palchik, Phys. Rep. 44 No. 5, (1978) 249.

[13] A.B. Zamolodchikov, Phys. Lett. B 97 (1980) 63.

[14] A.P. Isaev, Quantum groups and Yang-Baxter equations, Sov.J.Part.Nucl. 26 (1995) 501-526.

[15] B. Eden, C. Schubert and E. Sokatchev, Phys. Lett. B 482 (2000) 309, hepth/0003096;

B. Eden, P. S. Howe, C. Schubert, E. Sokatchev and P. C. West, Phys. Lett. B 466 (1999) 20, hep-th/9906051;

G. Arutyunov, B. Eden, A.C. Petkou and E. Sokatchev, Nucl. Phys. B 620 (2002) 380, hep-th/0103230.

[16] F. A. Dolan and H. Osborn, Nucl. Phys. B 629 (2002) 3, hep-th/0112251.

[17] G. t'Hooft, Nucl. Phys. B61 (1973) 455.

[18] S.G. Gorishnii and A.P. Isaev, Theor. Math. Phys. 62 (1985) 232. 
[19] D.J. Broadhurst, Z. Phys. C32 (1986) 249.

[20] D.T. Barfoot and D.J. Broadhurst, Z. Phys. C41 (1988) 81.

[21] D.Broadhurst, J.A. Gracey and D. Kreimer, Z. Phys. C 75 (1997) 559; hep-th/9607174.

[22] A.V. Kotikov, Phys. Lett. B 375 (1996) 240, hep-ph/9512270; The Gegenbauer Polynomial Technique: the evaluation of complicated Feynman Integrals, hep-th/0102177.

[23] N.I. Ussyukina and A.I. Davydychev, Phys. Lett. B 305 (1993) 136.

[24] D.J. Broadhurst, Phys. Lett. B 307 (1993) 132.

[25] N.I. Ussyukina and A.I. Davydychev, Phys. Lett. B 298 (1993) 363.

[26] N.I. Usyukina and A.I. Davydychev, Phys. Lett. B 332 (1994) 159, hep$\mathrm{ph} / 9402223$.

[27] A.I. Davydychev and J.B. Tausk, Phys. Rev. D 53 (1996) 7381, hep$\mathrm{ph} / 9504431$.

[28] V.A. Smirnov and O.L. Veretin, Nucl. Phys. B 566 (2000) 469, hep-ph/9907385.

[29] T. Gehrmann and E. Remiddi, Nucl. Phys. B 601 (2001) 248, hep-ph/0008287.

[30] C. Anastasiou, J.B. Tausk and M.E. Tejeda-Yeomans, Nucl. Phys. Proc. Suppl. 89 (2000) 262, hep-ph/0005328.

[31] K.G. Chetyrkin, A.L. Kataev and F.V. Tkachov, Nucl. Phys. B174 (1980) 345.

[32] S. E. Derkachov, G. P. Korchemsky and A. N. Manashov, Nucl. Phys. B 617 (2001) 375, hep-th/0107193.

[33] L.N. Lipatov, JETP Lett. 59 (1994) 596, hep-th/9311037; Phys. Lett. B 309 (1993) 394. 